1

2

3

4

5

6

7

8

9

10 9

\title{
Increased brain glucocorticoid actions following social defeat in rats facilitates the long-term establishment of social subordination
}

Meltem Weger ${ }^{\mathrm{a}}$, Yannick Sevelinges ${ }^{\mathrm{a}}$, Jocelyn Grosse ${ }^{\mathrm{a}}$, Isabelle Guillot de Suduiraut ${ }^{\mathrm{a}}$, Olivia Zanoletti $^{\mathrm{a}}$, and Carmen Sandi ${ }^{\mathrm{a}^{*}}$

${ }^{a}$ Laboratory of Behavioral Genetics, Brain Mind Institute, School of Life Sciences, École Polytechnique Fédérale de Lausanne (EPFL), 1015 Lausanne, Switzerland

*Corresponding author: $\underline{\text { carmen.sandi@epfl.ch }}$

Keywords: Corticosterone; social defeat; rats; dominance hierarchy; memory; lateral septum; medial amygdala; basolateral amygdala; nucleus accumbens. 
1 Abstract

2 Social rank is frequently established through aggressive encounters between new conspecifics. Despite 3 increasing evidence suggesting that social rank is critical for the well-being of both humans and 4 animals, knowledge about the factors influencing social rank remain scarce. Stress was previously 5 shown to affect the establishment and maintenance of social hierarchies in rats. Likewise, increasing 6 systemic corticosterone levels post-encounter in the emerging subordinate rat facilitates the long-term 7 establishment of social subordination. Here, we investigated whether central corticosterone actions are 8 sufficient to mediate this effect. Our data shows that, indeed, an intracerebroventricular corticosterone 9 injection given to the emerging subordinate rat facilitates the long-term maintenance of the subordinate rank. Next, we attempted to identify a particular brain region in which enhancement of corticosterone actions could be sufficient to exert the facilitation of a long-term maintenance in the emerging subordinate brain. However, post-encounter administration of corticosterone into the basolateral amygdala, medial amygdala, lateral septum and the nucleus accumbens, brain regions selected for their implication in social rank establishment and emotional modulation of memory, did not affect long-term social subordination. Our study highlights the involvement of intracerebral corticosterone actions on the facilitation of long-lasting subordinate behavior, likely by having a modulatory role in the neurobehavioral plasticity engaged in the shaping of social subordination. 


\section{Introduction}

Most social animal species are arranged in dominance hierarchies [1], which follow the organizing principle that dominant animals have priority access to important resources. Social rank is frequently determined by intrinsic factors (e.g., body size, body weight), sometimes including rank inheritance from parents. However, in many occasions encounters with new conspecifics lead to a newly established rank [2]. Once settled, social hierarchies tend to be long-lasting [2]. Despite the great impact of rank for health and well-being in both animals and humans [3,4], information about the factors and mechanisms affecting the long-term establishment of social hierarchies is still scarce.

Using rat as model system, we have previously shown that when a pair of unfamiliar male rats competes for the establishment of a social hierarchy, acute stress experienced by one of the two rats just before their encounter substantially increases the probability that the stressed rat becomes subordinate [5]. As each pair of rats is matched for all possible intrinsic factors (i.e., age, body weight and size, anxiety levels, and previous life conditions), this observation allows to establish a causal role for stress on the resulting social order. Importantly, the impact of stress goes beyond the actual outcome of the first contest between the competing animals. Thus, while the hierarchical relationship established under non-stress conditions is not observed when the same two animals are confronted again one week afterwards, in those dyads, in which rank was set involving one stressed rat, the same rank is observed again [5]. Administration of a protein synthesis inhibitor interfered with this potentiation of memory by stress, suggesting that a 'memory consolidation' process is involved in the long-term facilitation of the maintenance of the social hierarchy by stress [5].

Given that glucocorticoids, end products of the activated hypothalamus-pituitary-adrenal (HPA) axis, are activated by social encounters [6,7], elevated glucocorticoid levels at the time of a first social encounter between two individuals were hypothesized to affect the long-term establishment of social rank [8]. This hypothesis was based on two lines of evidence regarding glucocorticoid actions in brain and behavior. The first one relates to the ability of glucocorticoids to exert rapid, non-genomic effects in specific cell types and brain regions [9,10] swiftly resulting in behavioral activation [11-13] and enhancing aggression in ongoing contests [14]. The second one relates to a long-standing literature linking post-experience glucocorticoids with the facilitation of long-term memory [for reviews, see [15-18]]. Accordingly, our previous work showed that whereas systemic corticosterone treatment given to one of the two rat contenders before a first social encounter does not affect the outcome of the social hierarchy during a first encounter, it modified ongoing behavioral interactions when the injected male was the one that won dominance from the encounter [8]. However, the strongest effect was observed in the case when the subordinate rat received a systemic corticosterone injection immediately after the first social encounter. This treatment led to a long-lasting maintenance of the acquired social hierarchy [8], mimicking previously reported actions of stress in the same model [19]. In contrast, no long-term impact was observed when the dominant animal was the one that received the treatment [8]. Importantly, cumulative evidence in the literature indicates that once dominance relationships are established, plasma corticosterone swiftly normalize in the winner, while continuing elevated in the loser [[20-22]; for review, see [23]]. A key question arising from the findings implicating an enduring facilitation of the acquired subordination status by increasing post-encounter plasma corticosterone levels [8] is whether the effect could result from the central action of glucocorticoids.

Here, we asked whether we could exclude the necessity of peripheral glucocorticoid actions to account for the long-term facilitation of social subordination and, conversely, establish the sufficiency of brain corticosterone actions to induce this effect. To this end, we set a study to investigate whether intracerebroventricular (i.c.v.) administration of corticosterone to the animal emerging as subordinate 
out of a first dyadic contest would potentiate the long-term maintenance of the subordinate rank as well. As a secondary goal, we set to ask whether we could identify one specific region that would account for the corticosterone effect. Specifically, we performed experiments to assess whether postencounter administration of corticosterone into specific brain regions selected for their implication in social rank establishment and emotional modulation of memory (i.e., basolateral and medial amygdala nuclei, lateral septum and nucleus accumbens) would be able to facilitate long-term maintenance of subordination.

\section{Results}

Post-encounter intracerebroventricular administration of corticosterone facilitated the long-term maintenance of social subordination

Animals of each dyad underwent a first social encounter consisting of a social interaction test followed by a food competition test. Immediately afterwards, the loser animal received an i.c.v. infusion of corticosterone $[10 \mu \mathrm{g} / 3 \mu \mathrm{l}$; dose selected based on previously published work [24]], while the dominant animal was infused with vehicle (Figure 1A). After one week in which both animals were housed separately, the very same dyads were reunited in a neutral home cage and given a water competition test to assess for the maintenance of the social rank established during the first social encounter (Figure 1A).

As expected, in vehicle-injected rats the social hierarchy established on day 1 was not maintained across days $(\mathrm{t}(5)=0.075, p=0.94$; Figure 1A). However, the dyads in which the subordinate animal was i.c.v.-infused with corticosterone after the first social encounter had the very same animals showing subordinate behavior during the water competition test $(\mathrm{t}(6)=3.084, p=0.02$; Figure $1 \mathrm{~B})$. Thus, i.c.v. corticosterone infusion in recently defeated rats facilitated the transition of social subordination established at day 1 to its maintenance on day 8. These results indicate that corticosterone actions in the brain are crucially involved in the facilitation of a long-term memory of social subordination in rats.

Post-encounter administration of corticosterone into specific brain regions did not affect long-term social subordination

After observing that corticosterone actions in the brain are crucial for the formation of a long-term social memory in rats, we next tried to identify a particular brain region in which enhancement of corticosterone actions could be sufficient to exert the effect. We selected a few key brain areas known to play important roles in memory modulation and/or social behavior, including the basolateral amygdala, medial amygdala, lateral septum and nucleus accumbens. We applied the same experimental set-up as before with dominant animals receiving post-encounter infusion of vehicle and subordinate animals receiving post-counter infusion of corticosterone into the here examined brain regions (Figure 2A).

The first brain area of interest was the basolateral amygdala which has been described to play a role for memory modulation by glucocorticoids [16] and aggressiveness in social encounters in rats [25]. Thus, we assessed the long-term impact of intra-basolateral amygdala corticosterone infusions in social subordination. However, our results showed that initially subordinate animals on day 1 did not behave subordinate on day 8 , but had equal chances to become dominant or subordinate during the second social encounter $(\mathrm{t}(16)=1.036, p=0.32$; Figure $2 \mathrm{~B})$. Thus, we did not confirm a potential role for the basolateral amygdala on the corticosterone-induced enhancement of a long-term social subordination. 
Then, we tested the potential efficiency of corticosterone infusions into the medial amygdala, as this brain region has been implicated in social behaviors and dominance hierarchies [16,26-28]. However, intra-medial amygdala infusion of corticosterone of subordinate animals failed as well to facilitate long-term social subordination in the infused rats $(\mathrm{t}(7)=0.002, p=1.00$; Figure $2 \mathrm{C})$.

We next assessed the efficiency of corticosterone infusions into the lateral septum, a brain region repeatedly implicated in social interactions and aggression [16,29-33]. However, intra-lateral septum infusion of corticosterone into subordinate animals did not lead to a long-term establishment of social subordination $(\mathrm{t}(6)=0.878, p=0.41$; Figure 2D).

Finally, we tested the effect of corticosterone infusions in the nucleus accumbens, a brain region involved in the facilitation of memory by glucocorticoids [34] and the establishment of social hierarchies in rodents [35-37]. However, intra-nucleus accumbens infusion of corticosterone in the emerging subordinate animals did not facilitate their rank as subordinate animals on day $8(\mathrm{t}(9)=$ $0.376, p=0.72$; Figure 2E).

\section{Discussion}

Fighting a contest, which leads to a hierarchical organization, elicits increases in plasma corticosterone levels [6,7] that tend to remain higher in the loser than in the winner of the encounter [20-23]. Our previous work established that post-training administration of corticosterone to the loser of a dyadic competitive encounter facilitates the expression of subordinate status when the same rats are re-united in a competitive setting one week afterwards [8]. Given that this effect was induced by systemic corticosterone injections [8], here, we enquired whether it could be mediated by central, as opposed to simply peripheral and indirectly mediated, corticosterone effects. Our results show that central postencounter corticosterone actions, elicited by an i.c.v.-injection of corticosterone, facilitate the longterm establishment of the emerging subordinate rank.

These findings are in line with substantial evidence from the literature linking subordinate status with higher circulating levels of corticosterone, which has been particularly reported in suppressed subordinate animals by despotic alpha males [for review, see [38]]. However, it is important to note that the relationship between corticosterone levels and social rank is complex and seems to be highly dependent on social context. Higher corticosterone levels are typically observed in the group experiencing the highest social stress, which is normally the case for low ranking animals [39] particularly on the first few days of co-habitation [40,41]. In addition, subordinate males living in complex social hierarchies were reported to have higher plasma corticosterone levels than those living in pairs [38]. Furthermore, male rats that develop a context conditioning to social defeat were found to show enhanced corticosterone levels as well when exposed to the conditioning context [42]. These findings further indicate that the impact of a social defeat goes beyond the actual fighting event and that re-activation of a social conditioning of defeat can engage a corticosterone activation.

As opposed to rapid and immediate glucocorticoid actions that could be elicited by fast corticosterone elevation during the social encounter or by pre-encounter corticosterone treatment, the long-term nature of the post-encounter treatment effects that we have observed argues in favor of the engagement of genomic effects $[9,15,23]$. Genomic effects have been largely implicated in the long-term memory facilitation by glucocorticoids [15-18]. Therefore, a plausible explanation for our findings (i.e., that enhancing brain corticosterone actions promote long-term social subordination) is that they represent a facilitation of plasticity processes involved in neural adaptations prompted by social defeat [43]. Elevating corticosterone actions in the recently emerging 'subordinate' brain could allow potentiating plasticity processes primed by the defeat experience. This hypothesis is supported by previous data 
implicating protein synthesis processes in the long-term facilitation of aggressive behavior by corticosterone [44] and of social subordination by stress [5]. Moreover, this hypothesis aligns with findings that glucocorticoid effects are the result of their interaction with experience-induced activation of specific neuronal ensembles and activated molecular mechanisms [23]. For example, Mikics and colleagues [45] showed that whereas glucocorticoids have no effect on aggression under non-challenging situations, they stimulate aggressive behavior when animals are socially challenged. The fact that glucocorticoids potentiate mechanisms underlying memory of a defeat fits with the concept that these stress-related steroids potentiate cognitive processes and behavioral adaptations enabling to recognize potential dangers and respond accordingly [46]. However, an alternative explanation to our findings is that what was potentiated was not a specific memory for the particular defeat experience but, more generally, neural adaptations related to the losing experience leading to what is known as the "loser effect" [47-49]. This possibility is supported by evidence that brain corticosterone actions at the time of a first aggressive encounter can help perpetuating aggressive tendencies [50]. In our case, the perpetuation of the social hierarchy would not rely on its specific recognition of the dominant opponent but on its enhanced predisposition to display submissive behaviors in future encounters with conspecifics. Disentangling these alternative explanations (i.e., facilitation of a memory for a specific social hierarchy versus facilitation of a loser effect) would require further work. However, our earlier finding that the stress facilitates the hierarchy-linked recognition memory for "a particular individual" instead of rendering individuals subordinate against any other conspecific [5] argues in favor of the former explanation. It is also important to note that the loser effect might require repeated exposures to social defeat for the corresponding neurobehavioral adaptations to be enduringly established [47-49].

A secondary goal in our study was to attempt to identify a brain region in which local enhancement of corticosterone actions in emerging subordinate rats would recapitulate the facilitation of long-term subordination elicited by both systemic [8] and, as reported in the current study, i.c.v. injections. To this end, we selected brain regions that have been implicated in the emotional facilitation of memory by glucocorticoids [i.e., the basolateral amygdala [16] and the nucleus accumbens [34]] and/or in the neural adaptations related to rank attainment in social hierarchies [i.e., the basolateral amygdala [25], the medial amygdala [16,26-28], the lateral septum [16,29-33] and the nucleus accumbens [35-37]]. However, local micro-infusions of corticosterone into each of these brain regions in recently emerged subordinate male rats failed to facilitate the long-term transfer of the social hierarchy. Given that negative results do not allow formulating strong conclusions, these data leave open the potential effectiveness of intra-cerebral corticosterone treatment. It remains elusive whether effectiveness could emerge from a different corticosterone dose in any of the brain regions tested, from a combination of injections in different brain areas or from the targeting of additional brain regions (such as hippocampus, hypothalamic attack areas, or prefrontal cortex) not included here. Importantly, the fact that glucocorticoids coordinate and integrate actions throughout different brain areas and systems [51] argues in favor of the potential need to apply a multi-site injection approach to be able to mimic systemic and i.c.v. effects of corticosterone. However, the magnitude, effort and demand in terms of animals to follow such an approach is well beyond the possibilities and scope of the current study.

Importantly, our findings with i.c.v.-infusion of corticosterone further reinforce the view that strengthening subordination in the defeated animal is a sufficient condition to perpetuate the formerly established social hierarchy [8]. Furthermore, they are in line with the idea that subordination, a behavioral adaptation that helps reducing potentially dangerous physical interactions with dominant individuals, is at the core of the maintenance of a social hierarchy [52]. In addition, the ability of corticosterone to facilitate plasticity processes in emerging subordinates fits with a body of data indicating that although aggressive encounters are typically stressful for both contestants, following 
1 the encounter, plasma glucocorticoids tend to swiftly drop in winning animals, while they remain high 2 in the losers [23].

3 In conclusion, we present evidence that intracerebral actions of corticosterone in the emerging 4 subordinate brain facilitate the maintenance of subordinate behavior. These findings add to the 5 literature further evidence supporting a modulatory role of glucocorticoids in neurobehavioral 6 plasticity and strongly argue for a critical role of those processes in the shaping of social 7 subordination. 
2 The authors declare that the research was conducted in the absence of any commercial or financial 3 relationships that could be construed as a potential conflict of interest.

\section{Acknowledgements}

5 We would like to thank members of the Sandi lab for helpful discussions. We acknowledge funding by 6 the Swiss National Science Foundation (31003A-176206/1; and NCCR Synapsy, grant No. 51NF407 158776) and European Union's Seventh Framework Program for research, technological development 8 and demonstration under grant agreement no 603016 (MATRICS) (to CS). 
Material and Methods

\section{Animals}

Male Wistar rats (Charles River Laboratories, Lyon, France) with a body weight of 225-250 g upon arrival were individually housed with ad libitum access to food and water. We applied the same experimental conditions in previous studies addressed to analyzing social dominance and the establishment of social hierarchies in rats, and particularly previous observations implicating systemic corticosterone in this phenomenon [5,8]; applying the same procedure here was mandatory to allow for comparison. These conditions allow for recovery of the animals from the subsequent surgeries or prevention of cannula removal by cage mates during the time of experimentation. Animals were kept in a $12 \mathrm{~h} \mathrm{light/dark} \mathrm{cycle} \mathrm{with} \mathrm{lights} \mathrm{switched} \mathrm{on} \mathrm{at} \mathrm{7:00} \mathrm{am} \mathrm{and} \mathrm{a} \mathrm{constant} \mathrm{temperature} \mathrm{at} 22 \pm 2^{\circ} \mathrm{C}$. Surgery was performed on the animals a few days after arrival, but otherwise they were left undisturbed for one week after arrival before starting the experiments. Experiments were carried out in accordance with the European Communities Council Directive of $24^{\text {th }}$ November 1986 (86/609/EEC) and approved through a license issued by the Cantonal Veterinary Authorities (Vaud, Switzerland). All efforts were done to minimize animal number and suffering.

\section{Intracerebral cannulation surgery}

Animals were implanted with stainless steel guide cannulas targeting the examined brain areas. Animals were anesthetized by administrating ketamine $(70 \mathrm{mg} / \mathrm{kg})$ and xylazine $(6 \mathrm{mg} / \mathrm{kg})$ by intraperitoneal (i.p.) injection. Animals were placed in a stereotaxic apparatus (David Kopf Instruments, Tujunga, CA, USA) and small holes were drilled through the skull for bilateral placement of steel 23 gauge guide cannulae (Plastics One, Roanoke, VA, USA) fitted with a removable dummy cannula into the medial amygdala (MeA), basolateral amygdala (BLA), lateral septum (LS), nucleus accumbens (NAc), and monolaterally in the right or left hemisphere for (i.c.v) infusions. Coordinates were based on the atlas of Paxinos and Watson [53] and are taken from bregma (in mm) except the D.V. coordinate of the BLA, which was measured relative to the surface of the dura. The coordinates were as follows: NAc: A.P. +1.2 , M.L. \pm 1.5 , D.V. -6.50 ; for BLA: A.P. -2.8 , M.L. \pm 5.1 , D.V. -6.5 ; for MeA: A.P. -2.5 , M.L. \pm 3.3 , D.V. -6.9; for i.c.v.: A.P. -0.9, M.L. \pm 1.5 , D.V. -3.2 ; for LS: A.P. -0.2 M.L. \pm 0.75 , D.V. -3.7. Cannulae were fixed to the skull with two anchoring screws and Vetbond Tissue Adhesive glue (3M) and dental acrylic (Duralay 2244; Reliance, Worth, IL). After surgery, antisedan was injected (0.04 ml s.c., Pfizer, Switzerland) to reverse the anesthesia. After behavioral experiments animals were sacrificed by decapitation and correct cannula placements was routinely verified with Evans blue histology.

\section{Elevated Plus Maze}

Before the social hierarchy experiments, animals were habituated to human handling for two min per day for three consecutive days. Then, anxiety-related behavior of the animals was determined with the elevated plus maze (EPM). The EPM is elevated $65 \mathrm{~cm}$ above the floor and consists of two opposing open arms (45 x $10 \mathrm{~cm})$ and two opposing closed arms (45 x $10 \mathrm{~cm}$ with walls of $50 \mathrm{~cm}$ height) that extend from a central platform $(10 \times 10 \mathrm{~cm})$. Lighting was kept at 16-17 lx on the open arms and 5-6 $1 \mathrm{x}$ in the closed arms. For the test, animals were individually placed onto the central platform with its head facing the same closed arm and were allowed to explore the EPM for $5 \mathrm{~min}$. The behavior of the animals was video recorded and analyzed with the Ethovision 3.1.16 tracking system (Noldus IT, Netherlands). Time spent in open and closed arms was measured. 
Animals were distributed into dyads each consisting of two with each other unfamiliar rats that were matched in their body weight and anxiety levels. Animals in each dyad were matched for their anxiety levels in the EPM, as anxiety evaluated through this procedure was previously shown to have an effect on its own on the outcome of a social competition $[35,37]$. Thus, the rats in each dyad were considered equal in their probability $(=50 \%)$ to become dominant or subordinate during a social encounter. Having both animals matched for the same anxiety levels allows assessing the impact of corticosterone treatment in the outcome of the encounter for individuals' rank attainment. For distinguishing the animals within a dyad, animals were fur marked either on the back or on the neck before each social encounter. Therefore, the animals were prior habituated to the fur marking three consecutive days before the first social encounter.

\section{Behavioral tests}

\section{First social encounter: social interaction and food competition test}

The animals received eight Chocopop flakes (Kellogg`s, Switzerland) for two consecutive days to get used to the rewarding food. Then they were habituated to the experimental setup of the first social encounter, which consisted of a social interaction test and a food competition test. Therefore, each rat of a dyad was first placed individually into a clean home cage for $20 \mathrm{~min}$. Subsequently, the rat was transferred into a food competition box (a $40 \times 60 \mathrm{~cm}$ plastic box with $32 \mathrm{~cm}$ high walls, a removable extra wall placed $10 \mathrm{~cm}$ from one of the short sides, and a feeder on the opposite side containing eight Chocopop flakes) for $10 \mathrm{~min}$ for exploration. An exposure to the experimental setup was in this way repeated three consecutive days before the first social encounter.

On day 1 of the experiment, rats were submitted to a first encounter with their dyad partner for a social interaction test in a neutral home cage without food and water for $20 \mathrm{~min}$, followed by the food competition test in which the rats had to compete for eight Chocopops during $10 \mathrm{~min}$. By live video monitoring of the social interaction test, the behavior of the animals was analyzed during the social interaction test and dominance was assigned to the animal in the dyad expressing the most offensive behavior.["keep down" (pushing the opponent to the floor), "offensive up" (standing on the hind legs on upright position), and "lateral threat" (pushing/approaching the opponent showing its side with an arched back)] and causing the most defensive behavior ["defensive up" (standing on the hind legs in response to offensive upright), "submissive posture" (lying on the back), and "freezing" (immobility)] in its counterpart. Consequently, the animal expressing the least offensive behavior and causing the least expression of defensive behavior in its counterpart was identified as the subordinate animal of the dyad. Social dominance was estimated by summation of the total duration of offensive behaviors for each rat in the dyad. The food competition test was applied to maintain the initial study design [5] and was not used in this study for the determination of dominance, as it turned out to be not informative in previous experiments in which animals were exposed to glucocorticoid manipulations [8]. The behavior of the animals during the social interaction test and food competition test was in addition video recorded for later analysis. After the end of the food competition test, microinjection into the brain of the animals from each dyad were done as described under "Infusion procedure" and then animals were transferred back to their home cages and left undisturbed for one week. The habituation and the experiments for the first social encounter was performed between 8:00 am and 14:00 pm.

\section{Second social encounter: water competition test}

On day 8 of the experiment, a water competition test was done to test the memory of the established social hierarchy. Therefore, animals were first water deprived for a period of $6 \mathrm{~h}$ before the test (from 8:00 am to 14:00 pm) and then animals from the same dyad like in the first encounter were placed into 
a neutral home cage. After 2 min of habituation, a bottle of water was placed on the cage and the behavior of the animals during the following $10 \mathrm{~min}$ was recorded. Social dominance was determined by summation of the total duration of water consumption for each rat in the dyad. The water competition test was performed between 14:00 to 19:00 pm.

\section{Infusion procedure}

After the first social encounter, each animal in each dyad was infused to ensure that all animals are submitted to the same experience. Thereby, the animal of the dyad becoming dominant during the first social encounter received microinjections of vehicle (Saline) and the animal of the dyad becoming subordinate during the first social encounter received corticosterone (HBC complex Sigma-Aldrich, \#C174) into the brain region of interest. This experimental set-up was based on our previous observation that post-encounter Corticosterone i.p. into subordinate animals leads to the formation of a long-term memory of a social hierarchy [8]. During the infusion procedure, rats were gently restrained by hand and injection needles were inserted that extended $1 \mathrm{~mm}$ (in case of BLA, i.c.v. and MeA) or 2 $\mathrm{mm}$ (in case of LS and NAc) from the tip of the cannula. Infusion was done bilaterally during $1 \mathrm{~min}$ of constant flow. The injectors remained in place for an additional minute after infusion to enable proper diffusion. The injected volumes and concentrations of corticosterone were as follows: for i.c.v.: 10 $\mu \mathrm{g} / 3 \mu \mathrm{l}$; for MeA: $1 \mathrm{ng} / 0.2 \mu \mathrm{l}$; for BLA: $1 \mathrm{ng} / 0.2 \mu \mathrm{l}$; for NAc: $5 \mathrm{ng} / 0.3 \mu \mathrm{l}$; for LS: $5 \mathrm{ng} / 0.4 \mu \mathrm{l}$.

\section{Scoring of behavior and statistical analysis}

Behavior in all tests was video-recorded and scored blindly using The Observer (XT 11 Noldus IT, Netherlands). Behavioral analysis was done as previously reported [5,8]. Briefly, for the social interaction test the percentage of offensive behavior within each dyad was calculated as the ratio of the duration of the contestant showing offensive behavior ("lateral threat", "offensive up", and "keeping down") in each confronted pair and the total time of these offensive behaviors within the dyad. For the water competition test duration of water consumption was assessed. The percentage of offensive behavior was calculated as the ratio of the duration of the contestant's water consumption and total drinking time within a dyad. The labels "subordinate" and "dominant" animals are based on their status after the first social encounter and thus independent of their status as dominant or subordinate after the second social encounter. All results are expressed as the mean \pm the standard error of the mean (SEM). A paired two-tailed t-test was used to compare the percentage of offensive behaviors within a dyad of rats competing together. Data analysis was done with GraphPad Prism 7 (GraphPad Software, Inc.). Asterisks in the figures indicate $p$-values of $* p \leq 0.05, * * p \leq 0.01$ and $* * * p \leq 0.001$. 


\section{References}

1. van der Kooij MA, Sandi C: The genetics of social hierarchies. Curr Opin Behav Sci 2015 Apr;2:52-57.

2. Broom M: A unified model of dominance hierarchy formation and maintenance. J Theor Biol 2002 Nov;219:63-72.

3. Marmot MG: Status syndrome: a challenge to medicine. JAMA 2006 Mar;295:1304-7.

4. Sapolsky RM: Social Status and Health in Humans and Other Animals. Annu Rev Anthropol 2004 Oct;33:393-418.

5. Cordero MI, Sandi C: Stress amplifies memory for social hierarchy. Front Neurosci 2007 Nov; $1: 175-84$.

6. BRONSON FH, ELEFTHERIOU BE: ADRENAL RESPONSE TO FIGHTING IN MICE: SEPARATION OF PHYSICAL AND PSYCHOLOGICAL CAUSES. Science $1965 \mathrm{Feb}$ $5 ; 147: 627-8$.

7. Schuurman T: Hormonal correlates of agonistic behavior in adult male rats. Prog Brain Res 1980;53:415-20.

8. Timmer M, Sandi C: A role for glucocorticoids in the long-term establishment of a social hierarchy. Psychoneuroendocrinology 2010 Nov;35:1543-52.

9. de Kloet ER, Karst H, Joëls M: Corticosteroid hormones in the central stress response: quickand-slow. Front Neuroendocrinol 2008 May;29:268-72.

10. Groeneweg FL, Karst H, de Kloet ER, Joëls M: Rapid non-genomic effects of corticosteroids and their role in the central stress response. J Endocrinol 2011 May;209:153-67.

11. Sandi C, Venero C, Guaza C: Nitric oxide synthesis inhibitors prevent rapid behavioral effects of corticosterone in rats. Neuroendocrinology 1996 May;63:446-53.

12. Sandi C, Venero C, Guaza C: Novelty-related rapid locomotor effects of corticosterone in rats. Eur J Neurosci 1996 Apr;8:794-800.

13. Mikics E, Barsy B, Barsvári B, Haller J: Behavioral specificity of non-genomic glucocorticoid effects in rats: effects on risk assessment in the elevated plus-maze and the open-field. Horm Behav 2005 Aug;48:152-62.

14. Kruk MR, Halász J, Meelis W, Haller J: Fast positive feedback between the adrenocortical stress response and a brain mechanism involved in aggressive behavior. Behav Neurosci 2004 Oct;118:1062-70.

15. Sandi C: Glucocorticoids act on glutamatergic pathways to affect memory processes. Trends Neurosci 2011 Apr;34:165-76.

16. Roozendaal B, McGaugh JL: Memory modulation. Behav Neurosci 2011 Dec;125:797-824.

17. Finsterwald C, Alberini CM: Stress and glucocorticoid receptor-dependent mechanisms in long-term memory: from adaptive responses to psychopathologies. Neurobiol Learn Mem 2014 Jul;112:17-29.

18. de Quervain D, Schwabe L, Roozendaal B: Stress, glucocorticoids and memory: implications for treating fear-related disorders. Nat Rev Neurosci 2017;18:7-19.

19. Castro JE, Varea E, Márquez C, Cordero MI, Poirier G, Sandi C: Role of the amygdala in antidepressant effects on hippocampal cell proliferation and survival and on depression-like behavior in the rat. PLoS One 2010 Jan;5:e8618. 
20. Haller J, Kiem DT, Makara GB: The physiology of social conflict in rats: what is particularly stressful? Behav Neurosci 1996 Apr;110:353-9.

21. Akers KG, Yang Z, DelVecchio DP, Reeb BC, Romeo RD, McEwen BS, et al.: Social competitiveness and plasticity of neuroendocrine function in old age: influence of neonatal novelty exposure and maternal care reliability. PLoS One 2008 Jun;3:e2840.

22. Michopoulos V, Higgins M, Toufexis D, Wilson ME: Social subordination produces distinct stress-related phenotypes in female rhesus monkeys. Psychoneuroendocrinology 2012 Jul;37:1071-85.

23. Haller J: The glucocorticoid/aggression relationship in animals and humans: an analysis sensitive to behavioral characteristics, glucocorticoid secretion patterns, and neural mechanisms. Curr Top Behav Neurosci 2014;17:73-109.

24. Briski KP: Effects of peripheral versus central administration of the endogenous glucocorticoid, corticosterone, and the glucocorticoid receptor agonist, RU 28362, on LH release in male rats. Brain Res 1995 Jan;670:66-74.

25. Biro L, Toth M, Sipos E, Bruzsik B, Tulogdi A, Bendahan S, et al.: Structural and functional alterations in the prefrontal cortex after post-weaning social isolation: relationship with species-typical and deviant aggression. Brain Struct Funct 2017 May;222:1861-1875.

26. So N, Franks B, Lim S, Curley JP: A Social Network Approach Reveals Associations between Mouse Social Dominance and Brain Gene Expression. PLoS One 2015; 10:e0134509.

27. Holmes MM, Goldman BD, Forger NG: Social status and sex independently influence androgen receptor expression in the eusocial naked mole-rat brain. Horm Behav 2008 Aug;54:278-85.

28. Timmer M, Cordero MI, Sevelinges Y, Sandi C: Evidence for a role of oxytocin receptors in the long-term establishment of dominance hierarchies. Neuropsychopharmacology 2011 Oct;36:2349-56.

29. Veenema AH, Beiderbeck DI, Lukas M, Neumann ID: Distinct correlations of vasopressin release within the lateral septum and the bed nucleus of the stria terminalis with the display of intermale aggression. Horm Behav $2010 \mathrm{Jul} ; 58: 273-81$.

30. Lukas M, Bredewold R, Landgraf R, Neumann ID, Veenema AH: Early life stress impairs social recognition due to a blunted response of vasopressin release within the septum of adult male rats. Psychoneuroendocrinology 2011 Jul;36:843-53.

31. Clinard CT, Barnes AK, Adler SG, Cooper MA: Winning agonistic encounters increases testosterone and androgen receptor expression in Syrian hamsters. Horm Behav 2016 Nov;86:27-35.

32. Cordero MI, Just N, Poirier GL, Sandi C: Effects of paternal and peripubertal stress on aggression, anxiety, and metabolic alterations in the lateral septum. Eur Neuropsychopharmacol 2016 Feb;26:357-67.

33. Wong LC, Wang L, D'Amour JA, Yumita T, Chen G, Yamaguchi T, et al.: Effective Modulation of Male Aggression through Lateral Septum to Medial Hypothalamus Projection. Curr Biol 2016 Mar 7;26:593-604.

34. Roozendaal B, de Quervain DJ, Ferry B, Setlow B, McGaugh JL: Basolateral amygdalanucleus accumbens interactions in mediating glucocorticoid enhancement of memory consolidation. J Neurosci 2001 Apr;21:2518-25.

35. Hollis F, van der Kooij MA, Zanoletti O, Lozano L, Cantó C, Sandi C: Mitochondrial function in the brain links anxiety with social subordination. Proc Natl Acad Sci U S A 2015 
Dec;112:15486-91.

36. Larrieu T, Cherix A, Duque A, Rodrigues J, Lei H, Gruetter R, et al.: Hierarchical Status Predicts Behavioral Vulnerability and Nucleus Accumbens Metabolic Profile Following Chronic Social Defeat Stress. Curr Biol 2017 Jul;27:2202-2210.e4.

37. van der Kooij MA, Hollis F, Lozano L, Zalachoras I, Abad S, Zanoletti O, et al.: Diazepam actions in the VTA enhance social dominance and mitochondrial function in the nucleus accumbens by activation of dopamine D1 receptors. Mol Psychiatry 2017 Jul 20;

38. Williamson CM, Lee W, Romeo RD, Curley JP: Social context-dependent relationships between mouse dominance rank and plasma hormone levels. Physiol Behav 2017 Mar;171:110-119.

39. Sapolsky RM: Cortisol concentrations and the social significance of rank instability among wild baboons. Psychoneuroendocrinology 1992 Nov;17:701-9.

40. Bronson FH: Establishment of social rank among grouped male mice: relative effects on circulating FSH, LH, and corticosterone. Physiol Behav 1973 May;10:947-51.

41. Louch CD, Higginbotham M: The relation between social rank and plasma corticosterone levels in mice. Gen Comp Endocrinol 1967 Jun;8:441-4.

42. Razzoli M, Roncari E, Guidi A, Carboni L, Arban R, Gerrard P, et al.: Conditioning properties of social subordination in rats: behavioral and biochemical correlates of anxiety. Horm Behav 2006 Aug;50:245-51.

43. Hammels C, Pishva E, De Vry J, van den Hove DLA, Prickaerts J, van Winkel R, et al.: Defeat stress in rodents: From behavior to molecules. Neurosci Biobehav Rev 2015 Dec;59:111-40.

44. Mikics E, Kruk MR, Haller J: Genomic and non-genomic effects of glucocorticoids on aggressive behavior in male rats. Psychoneuroendocrinology 2004 Jun;29:618-35.

45. Mikics E, Barsy B, Haller J: The effect glucocorticoids on aggressiveness in established colonies of rats. Psychoneuroendocrinology $2007 \mathrm{Feb} ; 32: 160-70$.

46. Smith SM, Vale WW: The role of the hypothalamic-pituitary-adrenal axis in neuroendocrine responses to stress. Dialogues Clin Neurosci 2006;8:383-95.

47. Van de Poll NE, De Jonge F, Van Oyen HG, Van Pelt J: Aggressive behaviour in rats: Effects of winning or losing on subsequent aggressive interactions. Behav Processes 1982 Jun;7:14355.

48. Hsu, Wolf: The winner and loser effect: integrating multiple experiences. Anim Behav 1999 Apr;57:903-910.

49. Kloke V, Jansen F, Heiming RS, Palme R, Lesch K-P, Sachser N: The winner and loser effect, serotonin transporter genotype, and the display of offensive aggression. Physiol Behav $2011 \mathrm{Jul}$ 6;103:565-74.

50. Kruk MR, Haller J, Meelis W, de Kloet ER: Mineralocorticoid receptor blockade during a rat's first violent encounter inhibits its subsequent propensity for violence. Behav Neurosci 2013 Aug;127:505-14.

51. de Kloet ER: From receptor balance to rational glucocorticoid therapy. Endocrinology 2014 Aug;155:2754-69.

52. Summers CH, Forster GL, Korzan WJ, Watt MJ, Larson ET, Overli O, et al.: Dynamics and mechanics of social rank reversal. J Comp Physiol A Neuroethol Sens Neural Behav Physiol 2005 Mar; 191:241-52. 
1 53. Paxinos G, Watson C: The Rat Brain in Stereotaxic. Acad Press 6 Ed 2007;456.

2

3 
2 Figure 1. Post-encounter intracerebroventricular administration of corticosterone

3 (A) Schematic representation of the experimental protocol. Day 1: First social encounter consisting of 4 a 20 min social interaction test (SIT) followed by intracerebroventricular (i.c.v) infusion to each 5 animal of a dyad. Vehicle (saline) into dominant animals and corticosterone (CORT) into subordinate 6 animals. Day 8: Second social encounter consisting of a $10 \mathrm{~min}$ water competition test (WCT) to test 7 for social memory. Images of brain sections were adapted from the rat brain atlas [53]. (B, C) The 8 percentage of total offensive behavior (mean S.E.M.) between two opponents shown in SIT on day 1 9 and the WCT on day 8 in the post-social encounter injection groups. Post-social encounter vehicle for dominant, post-social encounter CORT for subordinate rat. (B) i.c.v.-infusion of vehicle (saline) (n = 6). (C) i.c.v.-infusion of $10 \mu \mathrm{g} / 3 \mu \mathrm{l} \mathrm{CORT}(\mathrm{n}=7)$. The status 'dom' (dominant) or 'sub' (subordinate) in the WCT refers to the status that was obtained during the SIT on day 1. Abbreviations: cc, corpus callosum; Lv, lateral ventricle. ${ }^{*} p \leq 0.05,{ }^{* *} p \leq 0.01$, and ${ }^{* * *} p \leq 0.001$.

Figure 2. Post-encounter administration of corticosterone into specific brain regions for memory modulation and social hierarchy establishment

(A) Schematic representation of the experimental protocol. Day 1: First social encounter consisting of a 20 min social interaction test (SIT) followed by infusions with each animal of a dyad into key brain areas linked with memory-consolidation and hierarchy attainment (for details, see text). Vehicle (saline) into dominant animals and corticosterone (CORT) into subordinate animals. Day 8: Second social encounter consisting of a 10 min water competition test (WCT) to test for social memory. Images of brain sections were adapted from the rat brain atlas [53]. (B-E) The percentage of total offensive behavior (mean S.E.M.) between two opponents shown in the SIT on day 1 and the WCT on day 8 in the post-social encounter injection groups. Post-social encounter vehicle for dominant, postsocial encounter corticosterone (CORT) for subordinate rat. (B) Intra-basolateral amygdala (BLA) infusion of $1 \mathrm{ng} / 0.2 \mu \mathrm{l}$ CORT $(\mathrm{n}=17)$. (C) Intra-mediolateral amygdala (MeA) infusion of $1 \mathrm{ng} / 0.2 \mu \mathrm{l}$ CORT $(n=8)$. (D) Intra-lateral septum (LS) infusion of $5 \mathrm{ng} / 0.4 \mu \mathrm{l}$ CORT $(\mathrm{n}=7)$. (E) Intra-nucleus accumbens (NAc) infusion of $5 \mathrm{ng} / 0.3 \mu \mathrm{CORT}(\mathrm{n}=10)$. The status 'dom' (dominant) or 'sub' (subordinate) in the WCT refers to the status that was obtained during the SIT on day 1. Abbreviations: cc, corpus callosum. ${ }^{*} p \leq 0.05,{ }^{* *} p \leq 0.01$, and ${ }^{* * *} p \leq 0.001$. 
A

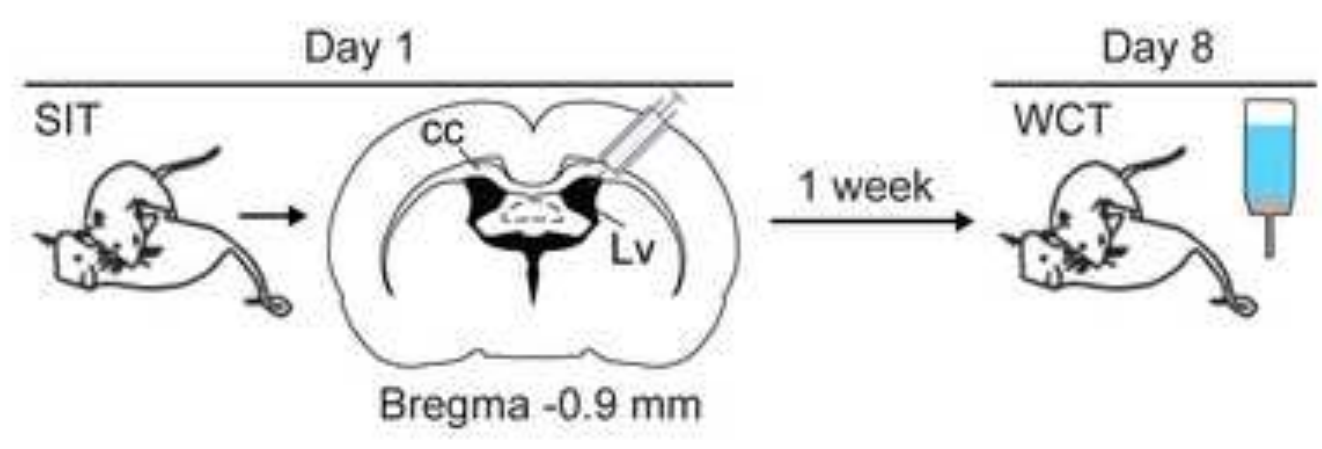

B

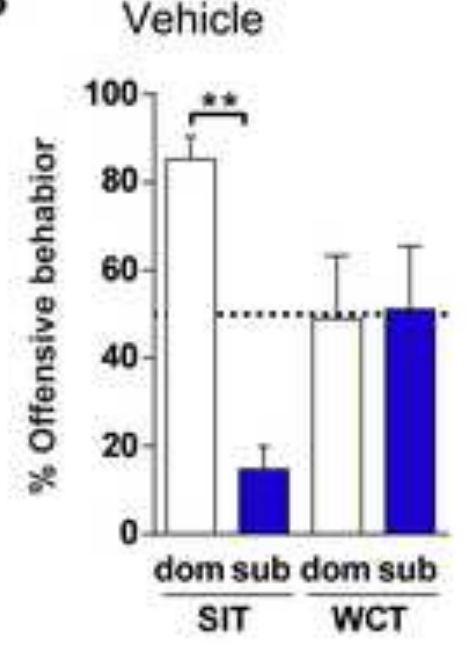

C

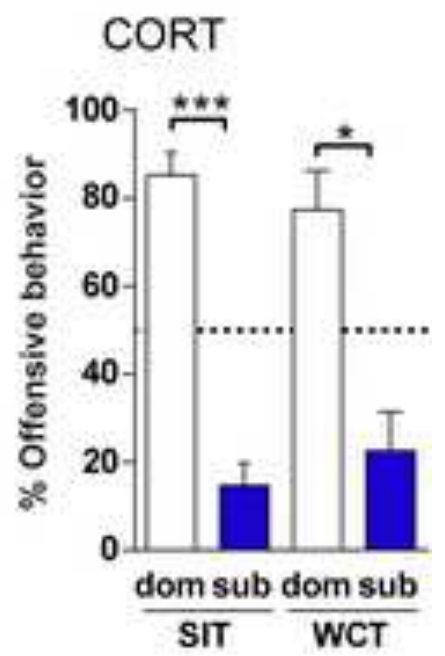


A

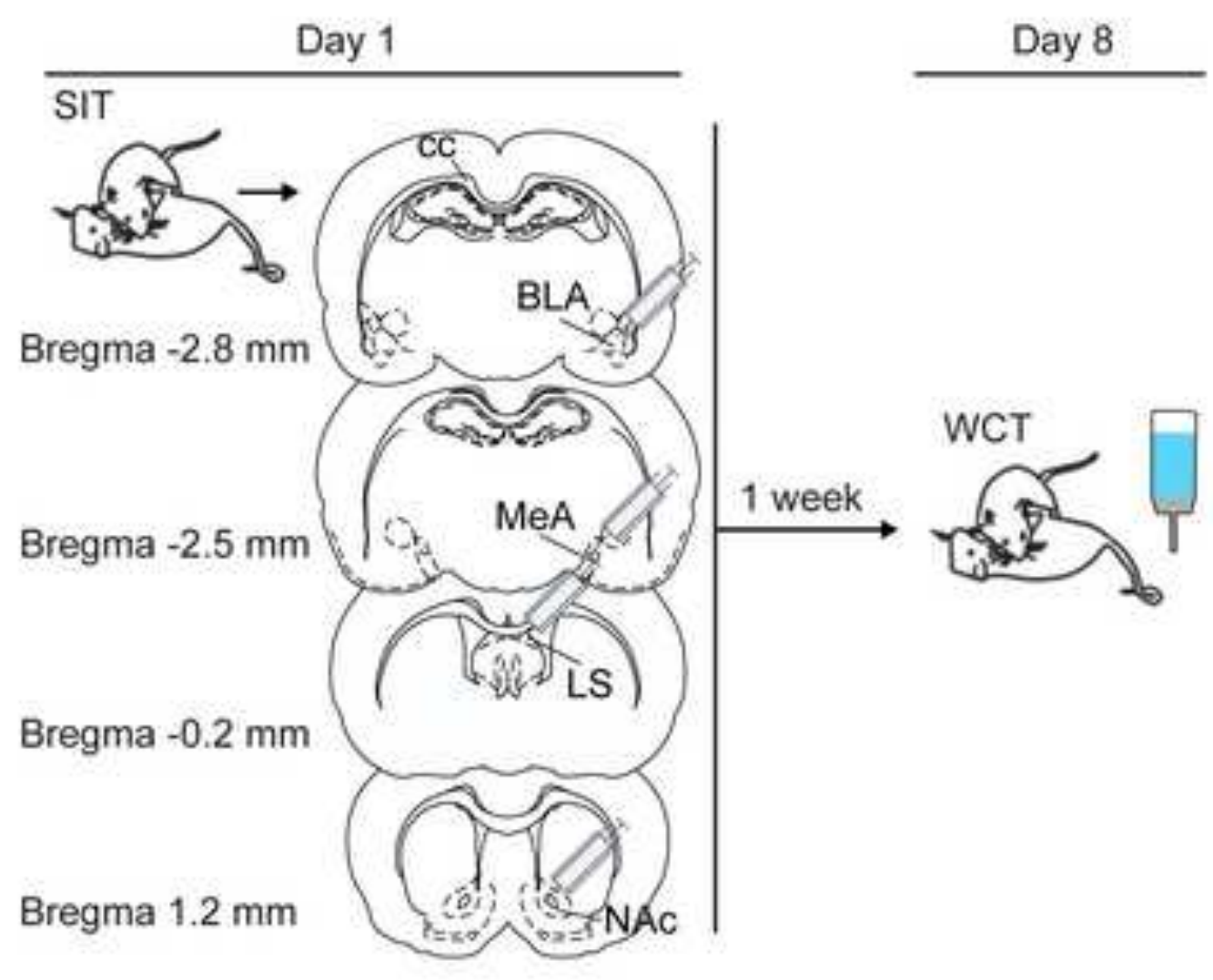

B

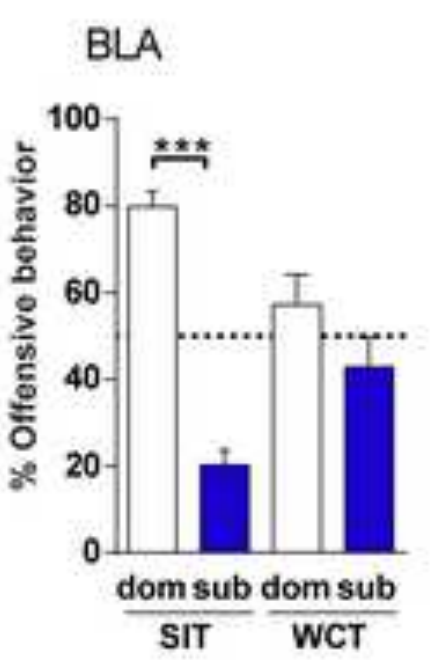

D

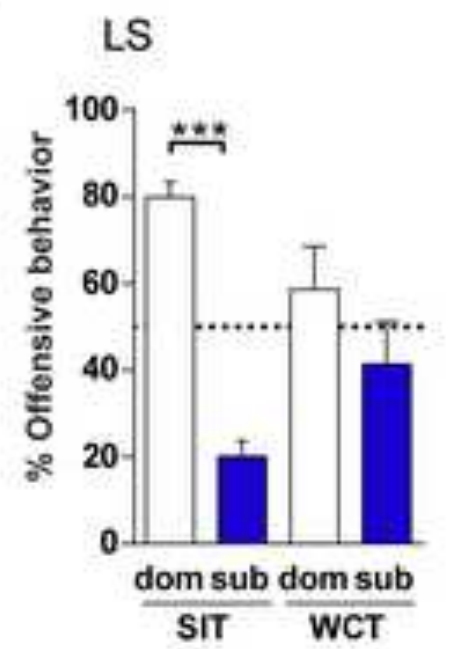

C

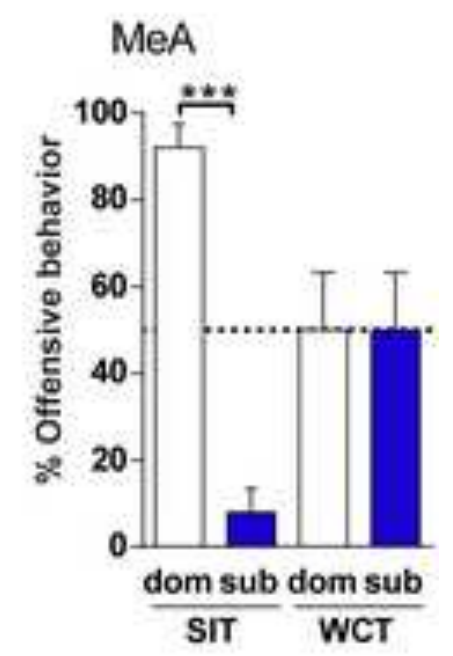

E

$$
\text { NAC }
$$

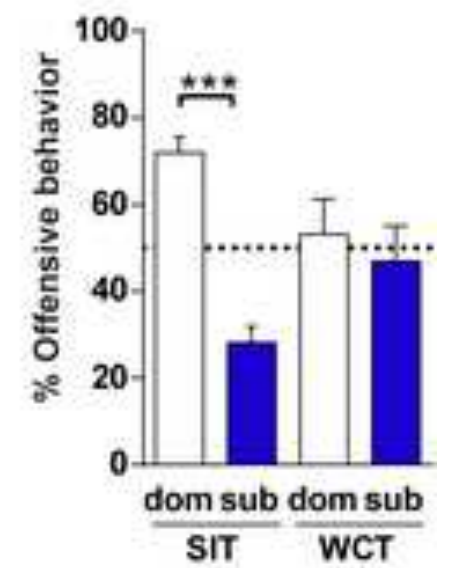

